\title{
ESTATUS DEL GÉNERO ACHROMOPORUS LOOMIS, 1936: ACTUALIZACIÓN TAXONÓMICA Y DE DISTRIBUCIÓN, CON NUEVOS REGISTROS DE LOCALIDADES
}

\begin{abstract}
Carlos Suriel
Museo Nacional de Historia Natural "Prof. Eugenio de Jesús Marcano" (MNHNSD). Calle César Nicolás Penson, Plaza de la Cultura Juan Pablo Duarte, Santo Domingo, República Dominicana. c.suriel@mnhn.gov.do
\end{abstract}

\section{RESUMEN}

Se hace una recopilación de la literatura referente al género Achromoporus Loomis, 1936, actualizando la información sobre su taxonomía, con la inclusión de los nuevos sinónimos y combinaciones de nombres; se ofrecen registros de nuevas localidades y los datos relativos al destino de los tipos.

Palabras clave: Achromoporus, recopilación, taxonomía, distribución, nuevos registros, destino de los tipos.

Title: Status of genus Achromoporus Loomis, 1936: taxonomic and distribution update with new records of localities.

\section{ABSTRACT}

A compilation of existing literature on the genus Achromoporus Loomis, 1936, was made, updating the information on its taxonomy, with the inclusion of new synonyms and name combinations; records of new localities and data concerning the fate of the types is included.

Key words: Achromoporus, compilation, taxonomy, distribution, new registers, destination types.

Harold Frederick Loomis describió el género Achromoporus con la especie tipo A. coloratus (1936: 112), en base a varios ejemplares machos y hembras procedentes de Thor, cerca de Puerto Príncipe, Haití; el holotipo fue depositado en U. S. National Museum of Natural History, Smithonian Institution (USNM) y los paratipos en Museum of Comparative Zoology, Harvard University (MCZ). En la misma publicación describió otras tres especies del género también procedentes de Haití y propuso una clave de identificación (1936: 113-19, Figs. 50-53). Posteriormente, describió A. heteromus para la República Dominicana en base a tres machos y una hembra procedentes de Sánchez, Samaná, colectados por P. J. Darlington, depositando los tipos en el MCZ (1941: 49-51, Fig. 17). El género Achromoporus se mantiene como un taxon endémico de la isla Hispaniola (Hoffman, 1979: 153; 1999: 268-69; ION, 2013; Pérez-Asso y Pérez-Gelabert, 2001: 73-74; Perez-Gelabert, 2008: 71).

Desde los trabajos de Loomis $(1936,1941)$, se han adicionado otras 18 especies nuevas para este género en la isla, todas de República Dominicana (Pérez-Asso, 2005, 2009; Suriel, 2010, 2011), haciendo un total de 23 , pero habría que restar la especie $A$. coloratus neiba, considerada posteriormente como especie no válida y sinónimo de A. coloratus Loomis, 1936 (Pérez-Asso, 2009). 
En su revisión del género, Pérez-Asso (2009) propone como sinónimo de Achromoporus a los géneros monotípicos Craterodesmus, Synecheporus, Biaporus y Lasiomazus, todos de la autoría de Loomis (1941). La razón fundamental para la propuesta de este arreglo es la estructura gonopodal con acropodito birramoso como rasgo común y distintivo de todos los demás géneros de milpiés de La Hispaniola, siendo Achromoporus el nombre más antiguo. Cada una de las especies tipo de estos cuatro géneros se incluyó en Achromoporus, pasando sus nombres a la condición de sinónimos nuevos y surgiendo cuatro nuevas combinaciones: A. concolor, A. montanus, A. ovatus y A. platyurus (Pérez-Asso, 2009); de esta manera, se agregan cuatro especies al género, totalizando 26 . El criterio del acropodito gonopodal dividido en dos ramas, propuesto por Pérez-Asso como rasgo definitorio del género, ha sido asumido posteriormente (Suriel, 2010).

La mayoría de las especies de Achromoporus están limitadas en su distribución a la localidad tipo (ver lista). De las cuatro especies descritas por Loomis para Haití, una ha sido registrada para República Dominicana: A. coloratus. Otras especies del género con nuevos registros de localidades que amplían el conocimiento de su distribución geográfica son: andujari, concolor, heteromus, magnus y occultus.

Para la recopilación e integración de la información actualizada sobre el género, se revisaron las publicaciones con todas las descripciones de especies de Achromoporus realizadas hasta el presente. Los nuevos registros de localidades partieron de la información asentada en el catálogo de la colección de diplópodos del Museo Nacional de Historia Natural "Prof. Eugenio de Jesús Marcano" (MNHNSD).

\section{LISTA DE ESPECIES DEL GÉNERO ACHROMOPORUS CON DATOS DE LA DESCRIPCIÓN, DESTINOS DE LOS TIPOS, SINONIMIAS Y ACTUALIZACIÓN DE SU DISTRIBUCIÓN GEOGRÁFICA}

Achromoporus coloratus Loomis, 1936. Especie tipo del género. Loomis, H. F. 1936. Bull. Mus. Comp. Zool., 80 (1): 113-15. Fig. 50. Holotipo: USNM. Paratipos: MCZ. Localidad tipo: Thor, cerca de Puerto Príncipe, Haití. Otras localidades en la descripción: Diquini, Carrefour y entre Petionville y Kenscoff, Haití. Registros adicionales (Pérez-Asso , 2009: 38): varias localidades en la Sierra de Neiba y al Norte de la Sierra de Bahoruco, República Dominicana; depositados en colección ARPA: 5365-66, 6158-6199, 6415-6461, 6561-6574. Un macho y una hembra donados a la colección MNHNSD por colección ARPA (6423 y 6440).

A. enneryensis Loomis, 1936. Loomis, H. F. 1936. Bull. Mus. Comp. Zool., 80 (1): 11516. Fig. 51. Holotipo: USNM. Paratipos: MCZ. Localidad tipo: Morne Pilboreau, Ennery, Haití.

A. furcipes Loomis, 1936. Loomis, H. F. 1936. Bull. Mus. Comp. Zool., 80 (1): 117-18. Fig. 52. Holotipo: USNM. Paratipos: MCZ. Localidad tipo: cerca de Plaisance, Haití. Otras localidades en la descripción: Morne Pilboreau, entre Plaisance y Ennery.

A. robustus Loomis, 1936. Loomis, H. F. 1936. Bull. Mus. Comp. Zool., 80 (1): 118-19. Fig. 53. Holotipo: USNM. Paratipos: MCZ. Localidad tipo: cerca de Cancoque, Haití. Otras localidades en la descripción: cerca de Plaisance, Haití.

A. heteromus Loomis, 1941. Loomis, H. F. 1941. Bull. Mus. Comp. Zool., 88 (2): 49-51. Fig. 17. Holotipo y paratipos: MCZ. Localidad tipo: Sánchez y alrededores, República Dominicana. No ha sido posible encontrar ejemplares de la especie, a pesar de mucha búsqueda, en toda la región de la localidad tipo. Nuevos registros: Los Jíbaros, San 
Cristóbal, República Dominicana (N 18-31.446, O 070- 16.831; 508 msnm), colección MNHNSD 19. 001; Majagual, San Cristóbal, República Dominicana (E 0371288 - N 2044130; E 371510 - N 2044198; 567 msnm), colección MNHNSD 19.017-19.021, 19.368-19.372, 19.1,553-19.1,564; Arroyo Grande, Los Cacaos, San Cristóbal, República Dominicana (E 0360691-N 2056228; E 360672-N 2056305, 593 msnm), colección MNHNSD 19.032, 19.1,565.

A. concolor (Loomis, 1941) comb. nov. Loomis, H. F. 1941. Bull. Mus. Comp. Zool., 88 (2): 52. Fig. 18, como Lasiomazus concolor. Holotipo y paratipos: MCZ. Localidad tipo: Loma Vieja, cerca de Constanza, Cordillera Central, República Dominicana. Pérez-Asso, A. R. 2009. Solenodon 8: 39-41. Figs. 2, 20 y 23: A. concolor (Loomis) comb. nov. Registros adicionales (Pérez-Asso, 2009: 39-41): cañón del río Bandera, cerca de la cabaña Fundación Moscoso Puello, Parque Nacional Valle Nuevo, La Vega, República Dominicana; depositados en colección ARPA: 8249-8253. Nuevos registros: Sabana Quéliz, Parque Nacional Valle Nuevo, República Dominicana, colección MNHNSD 19.1,671-1,674, 19.2,184-2,186.

A. montanus (Loomis, 1941) comb. nov. Loomis, H. F. 1941. Bull. Mus. Comp. Zool., 88 (2): 62-63. Fig. 23, como Biaporus montanus. Holotipo y paratipos: MCZ. Localidad tipo: Pico del Yaque, Loma Rucilla, Cordillera Central, República Dominicana. Otras localidades en la descripción: montañas al norte de Loma Rucilla. Pérez-Asso, A. R. 2009. Solenodon 8: 47-48. Figs. 6 y 23: A. montanus (Loomis), comb. nov. Solo conocida de las localidades registradas en la descripción.

A. ovatus (Loomis, 1941) comb. nov. Loomis, H. F. 1941. Bull. Mus. Comp. Zool., 88 (2): 60-61. Fig. 22, como Craterodesmus ovatus. Holotipo y paratipos: MCZ. Localidad tipo: Monte Diego de Ocampo, prov. Santiago (dato agregado), República Dominicana. Otras localidades en la descripción: Loma Quita Espuela, prov. Duarte (dato agregado). Pérez-Asso, A. R. 2009. Solenodon 8: 41-43. Figs. 3, 20 y 23: A. ovatus (Loomis) comb. nov., especímenes de Loma Quita Espuela depositados en colección ARPA: 4477-4486. Solo conocida de las localidades registradas en la descripción.

A. platyurus (Loomis, 1941) comb. nov. Loomis, H. F. 1941. Bull. Mus. Comp. Zool., 88 (2): 64-65. Fig. 24, como Synecheporus platyurus. Holotipo y paratipos: MCZ. Localidad tipo: Pico del Yaque, Loma Rucilla, República Dominicana. Pérez-Asso, A. R. 2009. Solenodon 8: 43-47. Figs. 4, 5, 20 y 23: A. platyurus (Loomis) comb. nov. Registros adicionales (Pérez-Asso, 2009: 44, 47): río Los Negros, Rincón de Piedra, San José de las Matas, prov. Santiago; sendero Los Tablones-Alto de la Cotorra, Cordillera Central, Parque Nacional Armando Bermúdez; localidades La Pirámide, Monumento a Caamaño (La Lechuguilla, Nizaíto, dato agregado) y camino entre La Pirámide y La Nuez, Parque Nacional Valle Nuevo. Especímenes depositados en colección ARPA: 5361-5362, 8691-8695, 8082-8157, 5842-5843, 7981-7999, 82338247; macho 8107 y hembra 8149 donados a la colección MNHNSD.

A. bayaguana Pérez-Asso, 2005. Pérez-Asso, A. R. 2005. Solenodon 5: 55-57; Figs. 2, 6, 10. Holotipo (3153), colección ARPA. Localidad tipo: Bayaguana, Monte Plata, República Dominicana.

A. ebanoverde Pérez-Asso, 2005. Pérez-Asso, A. R. 2005. Solenodon 5: 53-55; Figs. 1, 4-5, 10. Holotipo (3622), paratipos (3623-3627): colección ARPA. Localidad tipo: Loma de la Sal, Reserva Científica Ébano Verde, prov. La Vega, República Dominicana. Otras localidades en la descripción: Loma Casabito y El Arroyazo, Reserva Científica 
Ébano Verde, prov. La Vega, República Dominicana; especímenes depositados en colección ARPA, 3619, 3658-3659.

A. andujari Pérez-Asso, 2009. Pérez-Asso, A. R. 2009. Solenodon 8: 50-52; Figs. 8, 20, 23. Holotipo (8168), paratipos (8169-8186, 8187-8223): colección ARPA; paratipos 8179 y 8219 , donados a la colección MNHNSD. Localidad tipo: La Pirámide, Parque Nacional Valle Nuevo, República Dominicana. Nuevos registros: Sabana Queliz, Parque Nacional Valle Nuevo, República Dominicana (327711E, 2074936N), colección MNHNSD 19.1,597-1,611, 19.2,145-2,171; La Lechuguilla, Nizaíto, Parque Nacional Valle Nuevo, República Dominicana (331501E, 2070693N), colección MNHNSD 19.1,681-1,683, 19.2,131-2,141.

A. cachote Pérez-Asso, 2009. Pérez-Asso, A. R. 2009. Solenodon 8: 74-76; Figs. 19, 22, 23. Holotipo (6059), paratipos (6060-6067): colección ARPA. Localidad tipo: Cachote, camino de Ciénaga a Polo, prov. Barahona (dato agregado), República Dominicana.

A. chocoensis Pérez-Asso, 2009. Pérez-Asso, A. R. 2009. Solenodon 8: 63-65; Figs. 14, 21, 23. Holotipo (7318), paratipos (7319-7342), colección ARPA; paratipos, macho 7325 y hembra 7338 donados a la colección MNHNSD. Localidad tipo: área protegida El Choco, cerca del poblado de Cabarete, prov. Puerto Plata (dato agregado), República Dominicana.

A. isabelensis Pérez-Asso, 2009. Pérez-Asso, A. R. 2009. Solenodon 8: 68-69; Figs. 16, 21, 23. Holotipo (7471), paratipos (7469-7470): colección ARPA. Localidad tipo: Los Charcos del Damajagua, prov. Puerto Plata, República Dominicana; otras localidades de la descripción: base del Pico Gran Diablo, macizo montañoso Isabel de Torres, prov. Puerto Plata, República Dominicana.

A. jaragua Pérez-Asso, 2009. Pérez-Asso, A. R. 2009. Solenodon 8: 65-67; Figs. 15, 22 , 23. Holotipo (4631), paratipos (4680 y 4701): colección ARPA. Localidad tipo: La Curva del Guano, carretera de Oviedo a Pedernales, Parque Nacional Jaragua, prov. Pedernales, República Dominicana.

A. magnus Pérez-Asso, 2009. Pérez-Asso, A. R. 2009. Solenodon 8: 70-73; Figs. 17, 18, 21, 23. Holotipo (5810), paratipos $(5811-5817,8761-8780)$ y ejemplares adicionales asociados (8734-8750): colección ARPA. Localidad tipo: alrededores del poblado La Nuez, al sur del Parque Nacional Valle Nuevo, prov. San José de Ocoa (dato agregado), República Dominicana. Otras localidades de la descripción: Mata Grande y Sendero de Antón Sape Bueno a Antón Sape Malo, Parque Nacional Armando Bermúdez, prov. Santiago, República Dominicana. Nuevo registro: Alto de la Rosa, Parque Nacional José del Carmen Ramírez, prov. San Juan, República Dominicana.(1902'09”N, 071¹3’08’W), 1,744 msnm, colección MNHNSD 19.104.

A. martingarcia Pérez-Asso, 2009. Pérez-Asso, A. R. 2009. Solenodon 8: 61-63; Figs. 13, 21, 23. Holotipo (6709), paratipos (6710-6716): colección ARPA. Localidad tipo: cerca de Puerto Alejandro, Sierra Martín García, prov. Barahona, República Dominicana.

A. occultus Pérez-Asso, 2009. Pérez-Asso, A. R. 2009. Solenodon 8: 56-58; Figs. 11, 21, 23. Holotipo (8051) y paratipos (8052-8081, 5840-5841, 8158-8167, 8000-8009): colección ARPA. Paratipos, macho 8057 y hembra 8072 donados a la colección MNHNSD. Localidad tipo: La Pirámide, Parque Nacional Valle Nuevo, República Dominicana. Otras localidades de la descripción: Monumento a Caamaño (La Lechuguilla, Nizaíto, dato agregado), Parque Nacional Valle Nuevo. Nuevos registros: 
Sabana Quéliz (327786E-2074997N), Parque Nacional Valle Nuevo, La Vega, República Dominicana, colección MNHNSD 19.1,655-1,660.

A. pallidus Pérez-Asso, 2009. Pérez-Asso, A. R. 2009. Solenodon 8: 54-56; Figs. 10, 21, 23. Holotipo (5738), paratipos (5739-5745, 5747-5754, 5756): colección ARPA. Paratipos, macho 5746 y hembra 5755 donados a la colección MNHNSD. Localidad tipo: alrededores de La Pirámide, Parque Nacional Valle Nuevo, prov. La Vega, República Dominicana.

A. surieli Pérez-Asso, 2009. Pérez-Asso, A. R. 2009. Solenodon 8: 58-61; Figs. 12, 22 , 23. Holotipo (8696), paratipos (8697-8701, 8702-8711): colección ARPA. Paratipos, macho 8698 y hembra 8704 donados a la colección MNHNSD. Localidad tipo: La Ciénaga, sendero Los Tablones-Alto de la Cotorra, Parque Nacional Armando Bermúdez, prov. La Vega, República Dominicana. Otras localidades de la descripción en el Parque Nacional Armando Bermúdez: sendero Río Los Guanos, La Vega, y Antón Sape Malo, prov. Santiago.

A. vallenuevo Pérez-Asso, 2009. Pérez-Asso, A. R. 2009. Solenodon 8: 52-54; Figs. 9 , 20, 23. Holotipo (5726) y paratipos 5727-5737, 8010-8050, 5838-5839, 8229-8232: colección ARPA. Paratipos, macho 8026 y hembra 8048 donados a la colección MNHNSD. Localidad tipo: entre poblado La Nuez y la caseta del Parque Nacional Valle Nuevo (entrada al parque, dato agregado), prov. San José de Ocoa (dato corregido), República Dominicana.

A. atrechoensis Suriel, 2010. Suriel, C. 2010. Novitates Caribaea 3: 14-17; Fig. 1, A-G. Holotipo (19.562) y paratipos (19.484, 19.563, 19.559-19.561, 19.1,025, 19.1,026,19.1,031): colección MNHNSD. Paratipos, macho 19.560 y hembra 19.1,025 donados a la colección ARPA. Localidad tipo: mancha de bosque latifoliado entre pinares al pie de la loma El Atrecho, en el camino de Alto de la Rosa a Sabana Vieja: $19^{\circ} 02^{\prime} 54^{\prime \prime} \mathrm{N}, 071^{\circ} 12^{\prime} 49^{\prime}$ ' W, a 1,772 msnm, Parque Nacional José del Carmen Ramírez, prov. San Juan, República Dominicana. Otras localidades dentro del Parque José del Carmen Ramírez: Piedra del Aguacate y camino de Alto de la Rosa a Sabana Vieja. Simpátrica con A. elegans.

A. elegans Suriel, 2010. Suriel, C. 2010. Novitates Caribaea 3: 18-21; Fig. 2, A-G. Holotipo (19.485) y paratipos (19.554-19.555, 19.1,037-19.1,040, 19.962, 19.96419.965,19.1,115, 19.1,117): colección MNHNSD. Paratipos, macho 19.1,116 y hembra 19.1,118 donados a la colección ARPA. Localidad tipo: mancha de bosque entre pinares al pie de la loma El Atrecho, en el camino de Alto de la Rosa a Sabana Vieja: $19^{\circ} 02^{\prime}$ 54” N, $071^{\circ} 12^{\prime} 49^{\prime \prime}$ W, a 1,772 msnm, Parque Nacional José del Carmen Ramírez, prov. San Juan, República Dominicana. Otra localidad dentro del Parque Nacional José del Carmen Ramírez: Piedra del Aguacate. Simpátrica con A. atrechoensis.

A. bastardoae Suriel, 2011. Suriel, C. 2011. Novitates Caribaea 4: 22-26; Fig. 1, A-E. Holotipo $(19.1,590)$ y paratipos $(19.717-19.718,19.450,19.1,567,19.1,570-19.1,572$, 19.1,596, 19.1,584-19.1,588, 19.1,574-19.1,582, 19.1,587, 19.1,592-19.1,593, 19.1,595): colección MNHNSD. Paratipos, macho 19.1,585 y hembra 19.1,581 donados a la colección ARPA. Localidad tipo: camino de Las Yayitas a Cañaveral, entre los puntos 0358785E-2035014N y 359969E-2035734N, a 286 msnm, Las Yayitas, El Recodo, prov. Peravia, República Dominicana.

A. fractus Suriel, 2011. Suriel, C. 2011. Novitates Caribaea 4: 26-29; Fig. 2, A-E. Holotipo $(19.1,251)$ y paratipos $(19.1,252-19.1,253,19.1,255-19.1,256,19.1,261-19.1,262$, 
19.1,264, 19.1,267-19.1,269, 19.1,271, 19.1,347-19.1,349, 19.1,444): colección MNHNSD. Paratipos, macho 19.1,443 y hembra 19.1,263 donados a la colección ARPA. Localidad tipo: Loma Los Guayuyos, 0354037E-2036649N, a 1,359 msnm, Manaclar, prov. Peravia, República Dominicana; otra localidad en Manaclar: loma Los Pinos.

\section{AGRADECIMIENTOS}

Antonio R. Pérez-Asso hizo las sugerencias iniciales para la realización del trabajo, Gabriel de los Santos sugirió cambios pertinentes en la última versión y Katihuska Rodríguez colaboró en la curación y organización de especímenes en la colección de diplópodos del Museo Nacional de Historia Natural "Prof. Eugenio de Jesús Marcano"; a todos ellos mis agradecimientos.

\section{LITERATURA CITADA}

Hoffman, R. L. 1979. Clasification of the Diplopoda. Mem. Mus. His. Nat. Geneve. 209 pp.

Hoffman, R. L. 1999. Checklist of the millipedes of North and Middle America. Virg. Mus. Nat. Hist. Spec. Public. 8: 288 pp.

Loomis, H. F. 1936. The millipeds of Hispaniola, with descriptions of a new family, new genera, and new species. Bull. Mus. Comp. Zool. 80 (1): 3-197.

Loomis, H. F. 1941. Millipeds collected in Puerto Rico and the Dominican Republic by Dr. P. J. Darlington in 1938. Bull. Mus. Comp. Zool. 88 (2): 17-80.

Pérez-Asso, A. R. 2005. Dos especies y una subespecie nueva de milpiés del género Achromoporus (Diplopoda: Polydesmida) para Hispaniola. Solenodon 5: 53-59.

Pérez-Asso, A. R. 2009. El género Achromoporus (Diplopoda: Polydesmida: Chelodesmidae) en República Dominicana: especies nuevas y sinonimias. Solenodon 8: 33-81.

Pérez-Asso, A. R. y D. E. Pérez-Gelabert 2001. Checklist of the millipeds (Diplopoda) of Hispaniola. Boletín S.E.A. 28: 67-80.

Pérez-Gelabert, D. E. 2008. Arthropods of Hispaniola (Dominican Republic and Haiti): A checklist and bibliography. ZOOTAXA 1831, 71, pp. 530.

Suriel, C. 2010. Dos especies nuevas del género Achromoporus (Diplopoda: Polydesmida: Chelodesmidae) del Parque Nacional José del Carmen Ramírez. Novitates Caribaea 3: 13-21.

Suriel, C. 2011. Descripción de dos especies nuevas de Achromoporus (Diplopoda: Polydesmida: Chelodesmidae) para la República Dominicana. Novitates Caribaea 4: 21-30.

Thomson Reuters'Zoological [en línea]: Index to organism names (ION). 2013. <http://www. organismnames.com/query.htm?q.> [accesado: 21 de junio, 2012].

[Recibido: 05 de marzo, 2013. Aceptado para publicación: 06 de junio, 2013] 